ISSN 0511-5728

The West Indian Journal of Engineering Vol.44, No.1, July 2021, pp.18-25

\title{
Assessment of the Emerging Landscape of Unmanned Aerial Systems in Trinidad and Tobago
}

\author{
Raid Al-Tahir ${ }^{\mathrm{a}, \Psi}$, and Giatri K. Lalla ${ }^{\mathrm{b}}$ \\ Department of Geomatics Engineering and Land Management, The University of the West Indies, Trinidad and \\ Tobago, West Indies; \\ aEmail: raid.altahir@sta.uwi.edu \\ bEmail: giatri.lalla@my.uwi.edu \\ ${ }^{\Psi}$ Corresponding Author
}

(Received 10 June 2020; Revised 20 April 2021; Accepted 16 June 2021)

\begin{abstract}
Interest in the civilian applications of Unmanned Aerial Systems (UAS) has been growing worldwide, especially in government and commercial tasks such as surveillance, search and rescue, inspection of infrastructure, agriculture, mining, and mapping. Likewise, Trinidad and Tobago (T\&T) has been witnessing a growing interest and application of commercial and non-commercial UAS operations. However, there is little assessment for the growth of the UAS market nor is there characterisation of UAS-based activities since the pertinent regulations established in 2016. This study seeks to formally identify the emerging UAS landscape in T\&T during the period 2015 to 2019. As such, this study maps and characterises the spatial and temporal patterns of UAS distribution, then appraises the various categories for the existing operations. To achieve these goals, this study utilised qualitative and quantitative techniques of Geoinformatics. The intent for this study is to provide a perspective on the growth and the implications of the UAS industry in T\&T, and to guide strategic planning among organisations with a stake in the emergence of UAS into civil airspace.
\end{abstract}

Keywords: Unmanned Aerial System. Drone. Geoinformatics. Civil Aviation. Trinidad and Tobago

\section{Introduction}

Drones refer to aircraft without an on-board human pilot. These aircraft can be remotely controlled by a pilot on the ground or can fly autonomously based on preprogrammed flight plans aided by on board position and navigation sensors (Al-Tahir et al., 2011). While "drone" is the most popular term in the media, the professionally preferred terms are Unmanned Aerial Vehicle (UAV) and Unmanned Aerial System (UAS) (Fombuena, 2017; Granshaw, 2018). "UAS” is used to identify the actual air vehicle in addition to associated components (ground control station and the communication data link) that allow it to operate remotely. In a variation of this term, the letter " $A$ " refers to "aircraft" (Colomina and Molina, 2014; ICAO, 2011; TTCAA, 2016).

Unmanned aerial vehicles have been around since the early 1900s, when they were solely used for military operations. These vehicles were used as targets for antiaircraft training in the post-World War I era (late 1910's), and then as flying bombs during World War II (1940's). The actual armed use of the UAS started in the early 1990's when they were used for surveillance, and eventually in active combat missions in the beginning of the current century (Colomina and Molina, 2014; González-Jorge et al., 2017; Nguyen 2018).

Over the past two decades, the civilian use of unmanned aircraft has been expanding rapidly to support recreational and commercial applications. The increased demand for UAS is attributable to their low manufacturing and operational costs, as well as the flexibility of the platforms to accommodate the consumer's needs while eliminating the risk to pilots' lives in difficult missions (Al-Tahir and Arthur, 2012). Moreover, the continuous miniaturisation of electronics enabled the production of smaller, cheaper and more efficient UAS capable of being equipped with cameras and other sensors, hence provided greater availability (Floreano and Wood, 2015; Coops, Goodbody and Cao, 2019). The new technology offers the opportunity to replace or augment existing solutions and the expansion into new applications. By 2010, UAS production had more than doubled in 10 years while civilian and research applications had grown threefold and twofold, respectively (Al-Tahir and Arthur, 2012; van Blyenburgh, 2010). Furthermore, the UAS logistics and transportation market was estimated to be valued at US $\$ 11.20$ billion in 2022 and is projected to reach $\$ 29.06$ billion by 2027 (Murfin, 2018).

In Trinidad and Tobago (T\&T), the public's view of unmanned aircraft has significantly evolved over a number of years. This is due to the technological development, social influences, potential commercial use, and the easy access to the UAS. However, only few anecdotal indications are available for the early 
appearances of commercial UAS activities in the country prior to 2015. Specifically, there were few published academic research such as Al-Tahir et al. (2011) and Al-Tahir and Arthur (2012), as well as a number of newspaper articles on the use of drones for activities such as police operations (Persad, 2013). Another indication for early public interest is the tender for an UAS issued by the government to provide image products to satisfy the country's needs of spatial data (Colomina and Molina, 2014; GORTT, 2013).

On the other hand, the commercial and noncommercial UAS operations have grown continuously after 2015. For example, the Trinidad and Tobago Police Service (TTPS) was utilising UAS to monitor drug traffickers and other criminal activities (Alexander, 2016; Alonzo, 2018). Another example is the use of UAS for watching the Digity mud volcano (De Silva, 2018). More recently, a project for Birds Caribbean conducted UAS-based aerial surveys of specific high shorebird concentration sites in Trinidad to determine shorebird abundance and diversity and provide a base for effective shorebird management (Gebauer and Sorenson, 2020). Additionally, there are a large set of ongoing commercial applications and projects that do not get into the academic publications or the media.

It is clear that UAS are not only increasing in popularity, but are also rapidly being incorporated into different industries for commercial use. Against this backdrop of growing interest, this study aims to analyse the changing landscape of the UAS industry in T\&T in the period 2015-2019. The aim is to provide an important baseline for those interested in the UAS applications (academic, commercial, or otherwise) to understand the evolution of this industry in T\&T. This study firstly examines the spatial and temporal changes in the distribution of UAS using geoinformatics techniques. It then identifies the various categories, and assesses the spread of UAS users and operations in T\&T. Finally, the study concludes with identifying the existing and future implications of the growth of the UAS industry in T\&T.

\section{Regulations for the UAS Industry in T\&T}

The year 2015 witnessed a drastic increase in complaints by the citizens to TTPS against the use of UAS. The majority of the complaints revolved around the invasion of privacy and what was considered unfair competition in certain businesses (Alexander 2016). Consequently, Trinidad and Tobago's Civil Aviation Authority (TTCAA) established the Civil Aviation Unmanned Aircraft Systems Regulations (TTCAR No.19). These regulations were intended to address these issues by making it mandatory to register the UAS and obtain permission to operate in certain instances. One set of the regulations ensures the airworthiness of the system for operation, while another set focuses on physical and mental requirements for the operator. Another section provides regulations that disallow operating of a UAS with negligent or in an irresponsible manner that would endanger the life or the property of others.

Of specific interest to this study is a set of changes to the framework for the operation of the UAS that have influenced the growth of the UAS industry in T\&T. One of these changes dictates that UAS operators must keep an unaided visual line of sight of the aircraft. While a second observer can be utilised (without the aid of any device apart from corrective lenses), continuous communications must be maintained.

Another set of regulations prohibits the operation of UAS over persons not located under a covered structure that provides reasonable protection in the case of a system malfunction. As such, permission is needed for operating over private property such as land, vehicles, and all public properties. A verbal permission suffices to operate over private property if the aircraft is utilised for non-commercial or recreation purposes. Conversely, the UAS operator must acquire approval by the Civil Aviation Authority for commercial operations (where compensation is received for services provided). Approval for UAS operations in these situations is subject to providing proof of permission from each individual that would be present on the location.

The third subset of regulations that has influenced the growth of the UAS industry in the country is related to operating UAS over restricted areas. Specifically, UAS cannot be flown at an altitude above 121 meters (400 feet). Additionally, no operations are to be conducted within five kilometres $(\mathrm{km})$ from the boundary of any airport, within two km of any helipad, or within no fly zones specified by the Authority. Figure 1 represents the designated no fly zones and the navigation routes over T\&T.

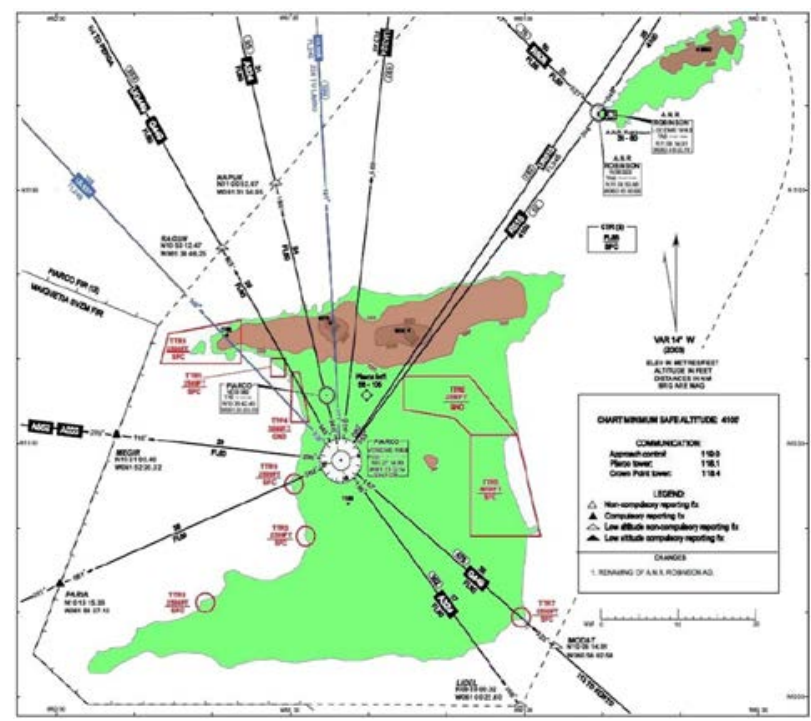

Figure 1. No Fly zones and navigation routes over Trinidad and Tobago (after Piarco FIR, 2020) 


\section{Data and Methodology}

Several datasets were utilised for the purpose of mapping and analysing the growth of the UAS industry in T\&T. The main set of data is UAS-specific dataset that was obtained from the Trinidad and Tobago Civil Aviation Authority (TTCAA). Ongoing since 2015, the TTCAA has been collecting data regarding the user operations and the distribution of the registered UAS in the country. Specifically, this dataset includes the following specific subsets:

o Registered UAS, which includes additionally the geographic locations of the UAS owners.

o UAS Characteristics; a non-spatial database describing the technical specifications for the registered UAS

o UAS Use; a non-spatial database that identifies the type and location of UAS-based operations in the country.

Other ancillary datasets were used in this study to provide the backdrop for the UAS data and to facilitate the intended analysis. These datasets were all GIS-ready and consisted of the following:

o Coastline, Regional Corporation Boundaries, and the Road Network for the country.

o Buildings dataset that depicts the location of all buildings in the country (as of 2007) and their categories (government, commercial, and residential).

o Restrictive Buildings dataset for structures and establishments that UAS flight restrictions may exist to protect them (e.g., Police Stations, and Hospitals, etc.).

o UAS No Fly Zones that delimits areas where UAS flights are restricted unless special approvals are granted as stipulated by the TTCAA Regulations.

Several spatial and statistical analysis tools within Geographic Information System (GIS) were utilised to extract spatial relationships that exist within the data. However, considering that the provided data were simple and limited, basic geoprocessing tools were suitable enough to produce the maps needed for this study. Given that two of the TTCAA datasets are non-spatial database, the first task was to unify and convert them into a spatial database. This was done using the Geocoding tool that would take a text-based address of the registered UAS operators and generate its coordinates within the national mapping coordinate system. Among the many records in this combined dataset, 750 records were then extracted exclusively for users who are citizens of, or residing in T\&T.

After combining the UAS datasets with the other ancillary datasets, fundamental GIS tools were used for performing queries, classifications and summarising a number of feature characteristics into attribute tables. Additionally, the Buffer operation was applied to determine which features would lie within the flying range of a UAS (for example, the typical range of a DJI drone is $5 \mathrm{~km}$ ). These features (structures and establishments) may be potentially exposed to physical harm due to drone operation or malfunction. As a result of this operation, there were many incidents of clustering of points that allowed for multiple buffers to be merged thus creating a larger buffer.

To infer any correlation or relationship, the several pertinent datasets must be overlaid with each other. Hence, the Overlay tool was utilised to perform operations such as point-in-polygon, line-in-polygon and polygon-on-polygon operations. Another tool utilised in this study was the density-based Clustering tool to identify specific areas where UAS points are concentrated and where they sparse. The result indicated the existence of points that clustered into a pattern using a minimum cluster of 25 . However, there were some data that did not cluster, these are usually singular events. This was completed by using the technique of unsupervised machine learning algorithm to detect the patterns.

Lastly, the geoprocessing tool known as a Directional Distribution (Standard Deviational Ellipse) was utilised. This tool was ideal for the statistical analysis of the data since it summarises the spatial characteristics of the point data by producing the central tendency, distribution and even the dispersion trends. When the Standard Deviational Ellipse tool is utilised, it creates an output class in the form of an elliptical polygon. The polygon uses the dataset's mean $\mathrm{X}$ and $\mathrm{Y}$ coordinates for the center, two standard distances (long and short axes), and the orientation of the ellipse.

\section{Analysing the Growth of UAS in T\&T}

\subsection{Spatial and Temporal Spread of the UAS in T\&T}

In order to examine the dynamics of the geographical spread of the UAS in the country, the following paragraphs look at the number, distribution, and type of the UAS devices in each year between 2015 and 2019. In 2015, there were only 39 UAS registered to individuals (personal use) although registration was voluntary. They were clustered around Port of Spain (the country's capital) and the lower Diego Martin, but sparsely scattered along the east-west corridor (see Figure 2). The majority of the UAS in the database consisted of quadcopters (four rotors) while there were three fixedwing UAS. About $85 \%$ of all the UAS were manufactured by DJI technology company such as the Phantom and Inspire series, although there were few UAS manufactured by Yuneec and DragonFly.

With the introduction of TTCAR No.19, the year 2016 witnessed a surge in the number of registered UAS in the country. By the end of the year, the number of registered UAS quadrupled with the addition of 178 devices, three of which registered in Tobago. As for the users, eight organisations and one club registered their UAS devices in that year. Figure 3 clearly indicates that registered UAS were clustered in urban areas on a much 


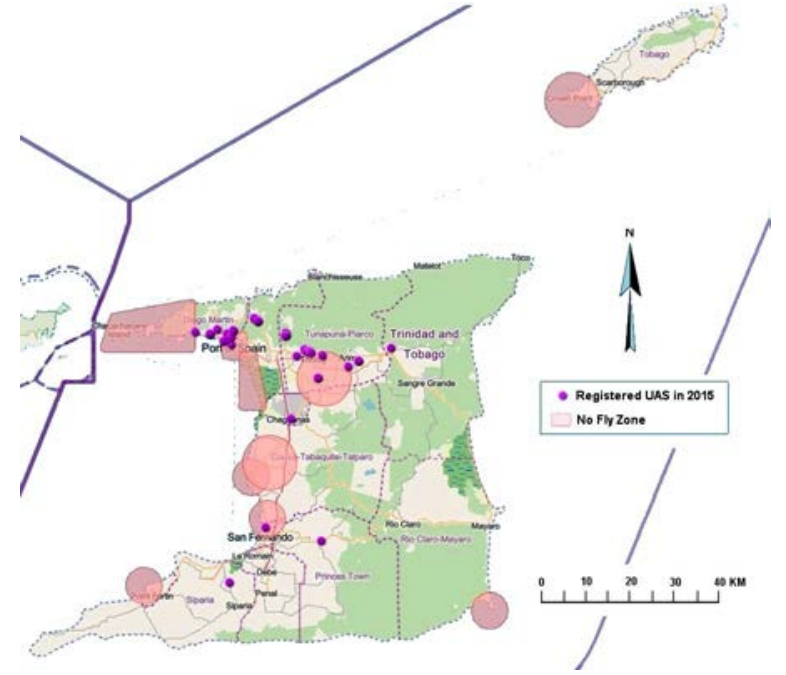

Figure 2. UAS distribution in 2015

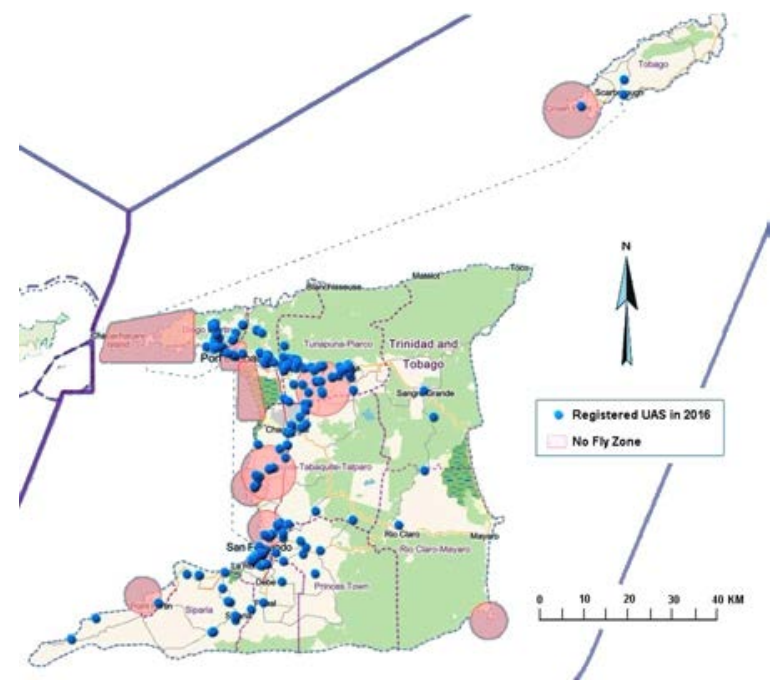

Figure 3. UAS distribution in 2016

larger scale than it had been in 2015. It can be noted that UAS were beginning to spread along the western side of the country. Quadcopters UAS were still dominating the UAS landscape in the year 2016, although there was an increase of four fixed wing UAS. While most of the UAS was still DJI Phantom, the database now included brands from other manufacturers such as Yuneec, Autel Robotics, HTEC, and MUVI.

In the year 2017, 166 registered UAS were added to the database including 22 UAS registered to organisations (nearly tripling the amount of registered organisational UAS). One can notice based on the distribution of the UAS in the country (see Figure 4) that the UAS industry was evolving to be more acceptable by the public. In addition to areas where they were previously existed, these systems expanded into new areas along the east-west corridor and in the cities of
Chaguanas and San Fernando which house the country's major industrial and commercial centres. As another noteworthy change in the industry, the DJI Phantom series was no longer the main type of UAS as DJI's Mavic Pro series became the most popular series in 2017.

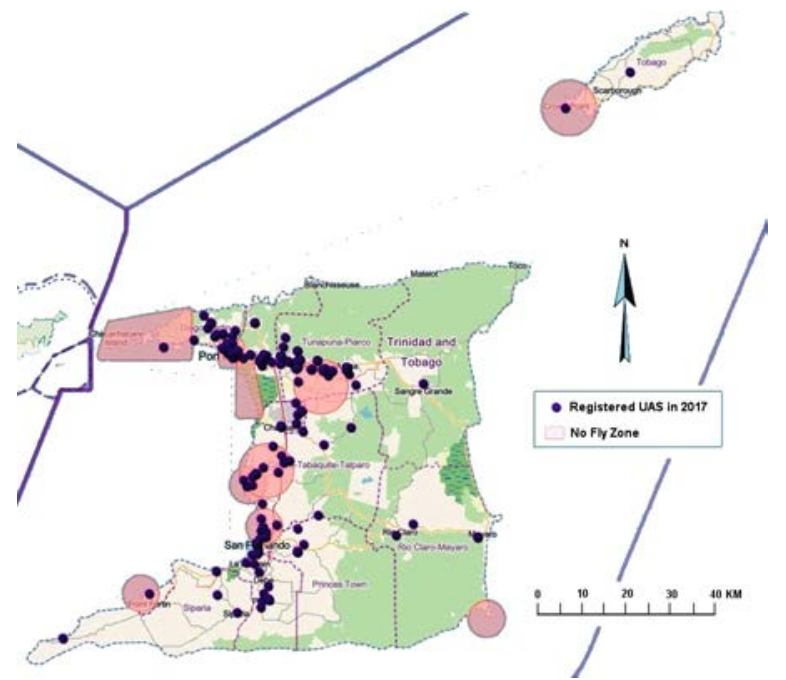

Figure 4. Distribution of UAS in 2017

The growth trend continued in 2018 and 2019 at almost the same pattern of distribution (see Figures 5 and 6) but at slightly declining numbers. There were 193 UAS added to the registry in 2018 and 154 UAS in 2019. These two years also witnessed the relatively significant proliferation of UAS in Tobago around the southwestern and the north-eastern sides of the island. The amount of registered organisational UAS steadily grew by 30 and 28 in 2018 and 2019, respectively. The majority of the UAS was still quadcopters, with an increase in the smaller size DJI Mavic series.

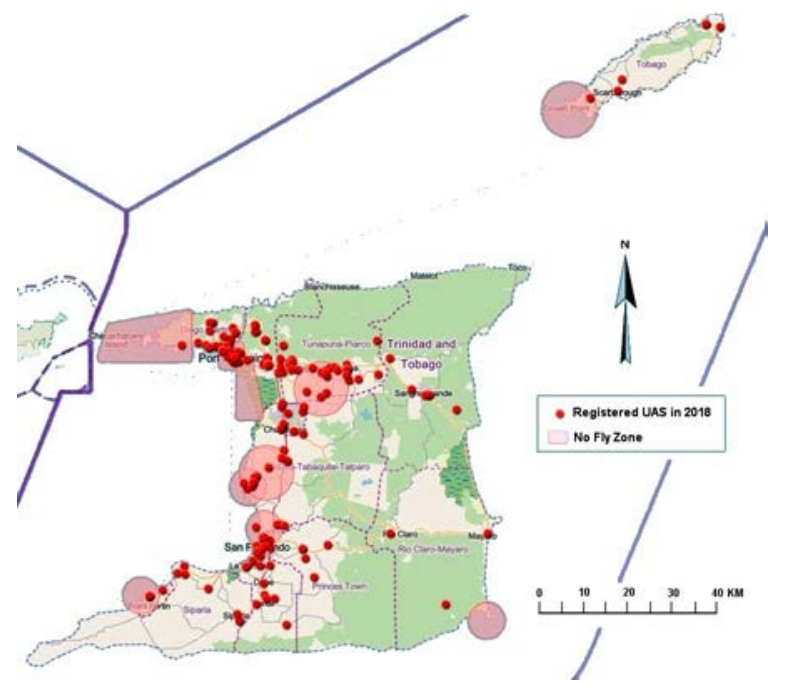

Figure 5. Distribution of UAS in 2018 


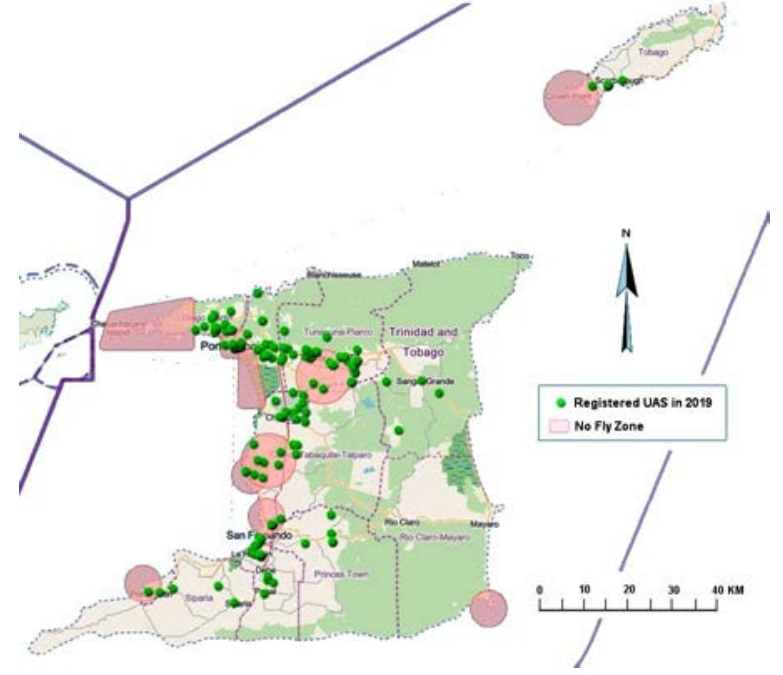

Figure 6. Distribution of UAS in 2019

At the same time, few numbers of hexacopters (six rotors) and octocopters (eight rotors) began to emerge in the registry. Table 1 provides a summary for the number of the UAS devices as categorised by the type of operators and by the UAS frame format.

Table 1. Summary characteristics of registered UAS in Trinidad and Tobago 2015-2019

\begin{tabular}{lll}
\hline \multirow{2}{*}{ Year } & UAS per Type of Operator & UAS per Type of \\
2015 & Personal: 39 & UAS Frame \\
2016 & Personal: 169, Organization: 8, Club: 1 & Fixed: 3, Quad: 36 \\
2017 & Personal: 144, Organization: 22 & Fixed: 4, Quad: 174 \\
2018 & Personal: 163, Organization: 30 & Fixed: 4, Quad: 187, \\
& & Hexa: 1, Octo: 1 \\
2019 & Personal: 126, Organization: 28 & Fixed: 4, Quad: 149, \\
\hline
\end{tabular}

Figure 7 presents an aggregate view of all registered UAS between 2015 and 2019. The figure asserts that most of UAS were clustered within the Port of Spain Regional Corporation, the Diego Martin Regional Corporation, and along the east-west corridor, mostly in Tunapuna Regional Corporation. Other three clusters can be noticed in the populated areas of Chaguanas, Couva, and San Fernando. One can also notice that the majority of the UAS expansion has been around commercial and industrial sites, and along the main road network of the country.

\subsection{Assessing UAS Operations in T\&T}

The TTCAA dataset catalogues all UAS-based operations and includes the type of operations and the number of approvals received against 25 recognised types of operations (see Figure 8). These operations can be further divided into three segments: government/public service, enterprise (commercial/ company/ organisation), and private operations.

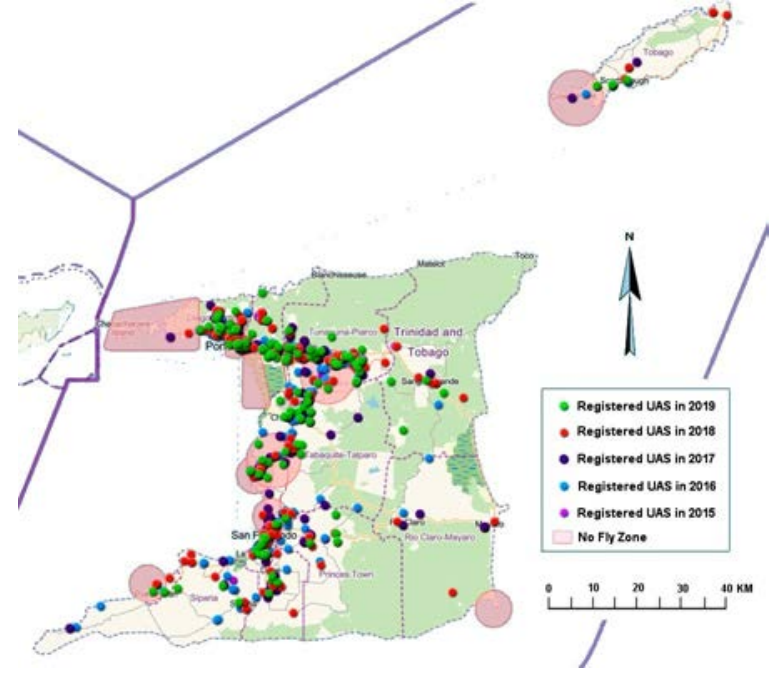

Figure 7. Distribution of all UAS registered in 2015 to 2019

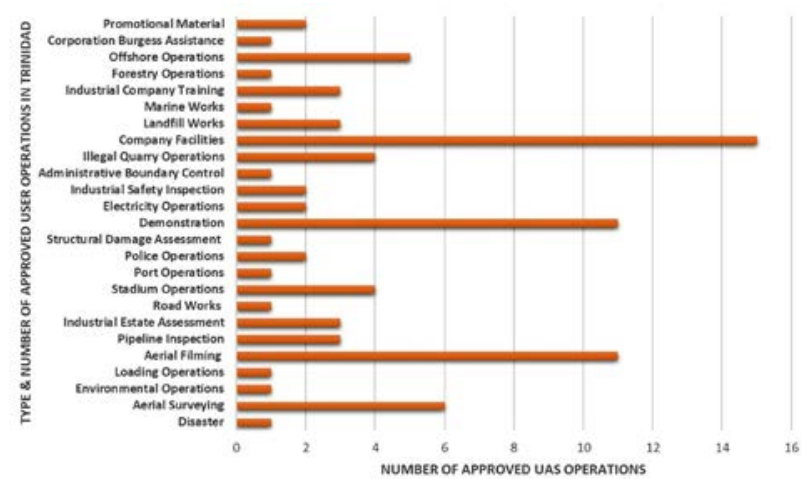

Figure 8. Type and number of approved operations in Trinidad and Tobago 2015-2019

The government/public service segment consisted of 17 approved operations completed by any public sector organisation in T\&T. The most prominent activities were related to quarrying and landfill operations. Other applications included disaster management and relief, corporation burgess, forestry, administrative boundary control, electricity works, police operations, and road works. Interestingly to note was that the number of approvals obtained for police operations was only two in total, which may indicate the existence of an arrangement between the Trinidad and Tobago Police Service (TTPS) and the TTCAA allowing the operations to take place without approvals.

The second segment of UAS operations in T\&T revolves around enterprise activities. There have been 32 approvals issued for this type of operations. Examples for such users are companies involved in the oil and gas industry as well as industrial facilities and services. The use of UAS is advantageous for the industrial companies (especially those with larger overall facilities) as it facilitates cost-effective, comprehensive, and in-depth levels of reconnaissance for wide areas. For example, 15 
UAS operations were specifically approved for the purpose of facilities management that were directed towards inspecting gas and pipeline leaks, roof leaks, building damages, faulty wiring, and similar aspects. The second largest activity in this segment was offshore work that accounted for five approvals. This included operations for safety training videos and offshore rig inspections since UAS is ideal for a quick visual overview and extensive surveying of hard to reach areas.

In the third segment, there were 37 UAS approvals for private UAS operations in T\&T since 2015. Aerial filming operations, with 11 approvals, had the lead in this segment. It encompasses a number of trades including movie productions, advertisements, and scientific operations. Equally in the lead in the approved private operations was the use of UAS for demonstration and promotional operations, a business that has seen a growth of UAS role fairly recently. Other activities in this segment included, though on a much smaller scale, operations related to marine, port, stadium, and loading operations.

Apart from the number and types of operations in Trinidad, it is also important to examine the pattern for where these operations took place. It is critical to remember that there are only 86 approvals being examined throughout Trinidad while only very few operations staged in Tobago. Therefore, the following inferences should be considered carefully regarding the spread of the operations throughout the island and offshore. Figure 9 undoubtedly indicates that the majority of the operations appear to be clustered at the south-western end of the Couva/Tabaquite/Talparo regional corporation.

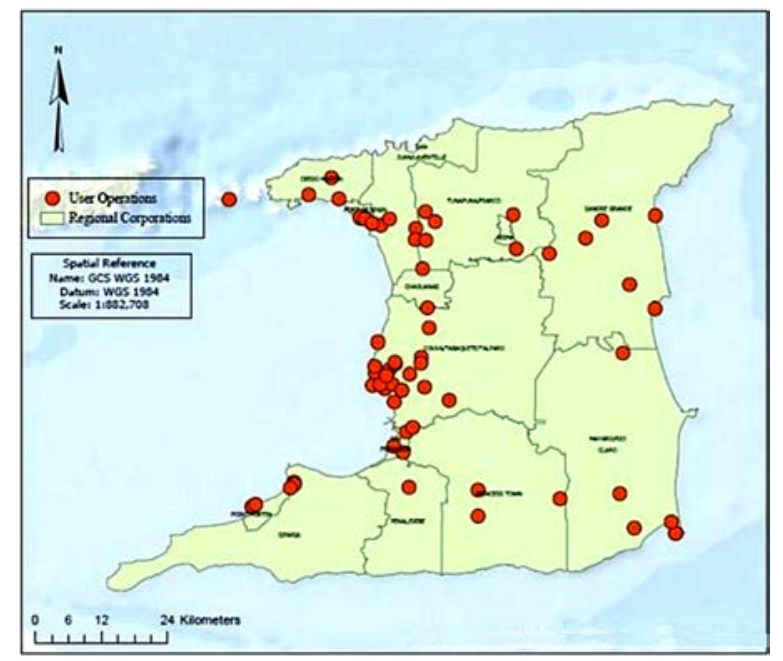

Figure 9. The distribution of UAS operations in Trinidad

This scenario could have been prompted by the nature of the operations in the area that ranges from facilities management to industrial operations, partly related to the activities of Point Lisas Industrial Estate.
Similar rationalisation may be the reason for the small number of operations around Point Fortin and Galeota where large oil estates are located. The second largest clustering was in Port of Spain and its vicinity. This can be attributed to the number of parks, buildings, and companies in addition to picturesque nature of the area. There was an interesting spread of individual operations across the east-west corridor and south-west to southeast corridor. This can be attributed to the type of commercial activities and UAS operations related to forestry and monitoring of quarrying operations.

\section{Analysis and Implications of the Growth of the UAS Industry in T\& T}

The previous section provided graphical presentations for the spatial patterns for the spread of the UAS in T\&T through the period 2015-2019. Based on that, key traits can be deduced regarding the changes in the UAS landscape over the period.

The registered unmanned aircraft are small or minisized devices that weigh less than 20 kilograms. These models are relatively easy to handle but are limited in flight range and time. The increase of the number of registered UAS reflects an increasing acceptance by the public and the users. One important observation is that setting up the Civil Aviation Unmanned Aircraft Systems Regulations had a significant positive impact in these aspects.

The growing number of the UAS and their operations is an indicative of the growing needs for such activities. The government has gradually increased the use of UAS in several of its operations together with the established use in homeland security and policing. For example, UAS were used to identify the affected areas and stranded residents during the floods of 2018 in Trinidad (Philip, 2018). Other examples include the government initiatives for utilising UAS for the mapping project mentioned earlier, and the recent efforts by the Ministry of Energy and Energy Industries for applying UAS technology to the monitoring, surveillance, and enforcement functions of the Minerals Division.

In parallel, the number of the commercial operations by enterprises and individuals can also be quite revealing about the growing operations in T\&T. Furthermore, some of the unmanned aircraft were solely registered for a specific use indicating more specialised and focused applications that require the technologically advanced UAS and personnel for the relevant operations.

Judging by the nature and growth of the UAS industry in T\&T, one may conclude key positive implications. Firstly, it is encouraging to observe that UAS users in all sectors are becoming more technologically advanced and by extension, the society as a whole. Secondly, the use of UAS will ultimately increase the efficiency and productivity of any project since fewer resources will have to be siphoned into operational costs. One final positive implication is that 
employing such technology induces further research and development for advancing the discipline and generating new knowledge.

On the other hand, there are also several negative implications. With the exponential increase of UAS in $\mathrm{T} \& \mathrm{~T}$, the possibility for a collision within the airspace or crash on the ground is exacerbated. This is a serious concern judging by the civilian air navigation routes (see Figure 1). While there are set regulations in place to ensure the airspace safety, there is no systematic mechanism for monitoring or policing that would guarantee compliance.

Another negative implication is the looming public safety and privacy concerns. The figures in the previous section clearly indicate that most of the registered UAS are located within urban areas and operate within the range of many public and private properties. Such a condition would most certainly lead to instances where citizens' privacy or safety is jeopardised. While many UAS operators have understood the cause for these concerns, many operators may be less caring while operating the UAS or may not seek permission to fly over a property as required by the regulations.

To address these problems and the negative implications, this study suggests the following recommendations towards the improvement of the regulations.

1. Promoting the use of relevant checklists by the operators to help in pre-flight inspection, as well as promoting the documentation of maintenance logs.

2. Issuing additional set of regulations to mandate reporting, site visits and monitoring the operations as a means of ensuring compliance.

3. Naming the exact operations that are not allowed over persons.

4. Setting up a dedicated entity to process permission requests so that individuals will not be deterred from the process due to its current excessive length.

It is also envisaged that the regulations should be updated to accommodate for the advancements in the unmanned aircraft that aim at improving their efficiency and preparing for their safe integration in the national airspace. Some of these intensely pursued subsystems are the Beyond Visual Line of Sight (BVLOS) that eliminates the need for direct visual monitoring by human, 'Detect and Avoid' that allows UAS to detect and safely steer clear of aircraft or other obstructions, and Geo-fencing for creating virtual fences around areas or points of interest to keep UAS away.

\section{Conclusion}

Over the last couple of decades, the world has experienced a growing utilisation of unmanned aerial systems to perform a broad spectrum of applications. The rising demand is influenced by the unique range of features that these aircraft offer along with the continuous advances in automation and sensor technologies. The emergence of a robust government and commercial UAS industry in $\mathrm{T} \& \mathrm{~T}$ is inevitable following the global trend. It is clear that civilian applications of UAS have been rapidly evolving, matched by their growing affordability and the advancement of the associated hardware and processing software. Scaling up the use of small drones from a niche market to widespread use in civilian applications depends on having appropriate regulatory frameworks in place. To understand the impact of the Civil Aviation Unmanned Aircraft Systems Regulations of 2016 and their future implications, this study analysed the growth of the UAS industry over the period 2015-2019. Geoinformatics tools were employed for this task using user information, the distribution of UAS, and the related operations.

Although there was a limited amount of data available to this study, it can be observed that the number and types of unmanned aircraft are rapidly increasing. As for the spatial patterns, one can recognise that UAS owners and their operations tend to cluster around the south-western end of the Couva/Tabaquite/Talparo corporation and Port of Spain city corporation. This is expected when one considers the concentration of business establishments and industrial opportunities at both locations.

The study identifies positive impacts for the UAS growth on the country manifested by the foreseen technological development, advancement of knowledge and research, and increase in the efficiency of commercial operations. Equally, the study envisages negative implications such as the airspace proliferations, general safety and privacy concerns. While there are set regulations to ameliorate these potential problems, there is a need to maintain, enforce, and update these regulations.

It is evident that large volumes of UAS are expected to be flying in the future, so safe operation is essential for the user and the public in general. As such, before the full potential of this market can be exploited the regulatory framework should respond to such needs and to evolving technological developments. Specifically, in response to the demand for autonomous operational capabilities, two related issues need to be addressed: the full integration of the UAS into the national airspace system and the removal of the legal restriction to fly beyond the operator's visual line of sight (BVLOS). An unmanned aircraft being operated BVLOS no longer has the protection of the pilot or observer to avoid terrain, obstacles or other aircraft.

Finally, the growth and future implications of the UAS industry in T\&T despite limitation in the pertinent regulation has potential to speak into policy that will streamline more founded regulations in the future. Other countries experiencing similar landscape may borrow from the experience of the study area. Further, considering the limited human and financial resources in the region, one recommendation might be to encourage 
more collaboration between Industry and Academia if this is not already the case.

\section{References:}

Alexander, G. (2016), "Drone worries for national security”, The Guardian, 30 January 2016, available at: http://www.classifieds.guardian.co.tt/news/2016-01-30/droneworries-national-security.

Alonzo, R. (2018), “Cops use drone to capture gunmen”, The Guardian, 6 January 2018, available at: http://www.classifieds.guardian.co.tt/news/2018-01-05/cops-usedrone-capture-gunmen.

Al-Tahir, R. and Arthur, M. (2012), "Unmanned aerial mapping solution for small island developing states", Proceedings of the Global Geospatial Conference (GSDI 13), Québec City, Canada, 14-17 May, 9 pages.

Al-Tahir, R., Arthur, M., and Davis, D. (2011), "Low cost aerial mapping alternatives for natural disasters in the Caribbean”, FIG Working Week, Morocco.

Colomina, I., and Molina, P. (2014), “Unmanned aerial systems for photogrammetry and remote sensing: A review", ISPRS Journal of Photogrammetry and Remote Sensing, Vol.92, pp.79-97.

Coops, N., Goodbody, T., and Cao, L. (2019), "Four steps to extend drone use in research", Nature, Vol.572 pp.433-435.

De Silva, R. (2018), "Digity mud volcano under watch", The Guardian, 15 February 2018, available at: http://www.classifieds.guardian.co.tt/news/2018-02-15/digitymud-volcano-under-watch.

Floreano, D., and Wood, R. (2015), "Science, technology and the future of small autonomous drones”, Nature, Vol.521, pp.460466. doi:10.1038/nature 14542 .

Fombuena, A. (2017), "Unmanned aerial vehicles and spatial thinking”, IEEE Geoscience and Remote Sensing Magazine, Vol.5, No.3, pp.8-18.

Gebauer, M. and Sorenson, L. (2020), Final Report: Shorebird Distribution, Abundance and Diversity in Trinidad-2018 to 2020, Gebauer \& Associates Ltd.

González-Jorge, H., Martínez-Sánchez, J., Bueno, M., and Arias, A.P. (2017), "Unmanned aerial systems for civil applications: A review”, Drones, Vol.1, No.2.; doi:10.3390/drones1010002.

GORTT (2013), Supply and Installation of an Unmanned Aerial Vehicle Mapping System and Terrestrial Laser Scanner for the Division of Lands and Surveys, Technical Report, Ministry of Housing, Government of the Republic of Trinidad and Tobago.

Granshaw, S. (2018), "RPV, UAV, UAS, RPAS... or just Drone?” The Photogrammetric Record, Vol.33, No.162, pp.160-170. DOI: $10.1111 /$ phor.12244.

ICAO (2011), Unmanned Aircraft Systems (UAS), ICAO Cir 328, International Civil Aviation Organisation, available at: http://www.icao.int/Meetings/UAS/Documents/Circular 328_en.pdf.
Murfin, T. (2018), "UAV Report: Growth Trends \& Opportunities for 2019". Special section, GPS World.

Nguyen, T. (2018), "The history of drones", available at: http://www.thoughtco.com/history-of-drones-4108018.

Persad, P. (2013), “Using drones in crime fighting”, The Guardian, 17 March 2013, available at: http://www.classifieds.guardian.co.tt/columnist/2013-0318/using-drones-crime-fighting.

Philip, D. (2018), “T\&T under Water”, Daily Express, October 20, 2018 available https://trinidadexpress.com/multimedia/photos/t-t-underwater/collection_a0f07adc-d4b7-11e8-891b-13d8854bc5b4.html.

Piarco FIR (2020), Eastern Caribbean Piarco Virtual FIR, Piarco Flight Information Region, Trinidad, available at: https://piarco.vatcar.org/downloads/TMA/PIARCO_TMA.pdf.

TTCAA (2016), "Civil Aviation [(No. 19) Unmanned Aircraft Systems] Regulations”, Trinidad and Tobago Civil Aviation Authority (available http://www.ttparliament.org/documents/2533.pdf.

van Blyenburgh, P. (2010) (ed), Unmanned Aircraft Systems: The Global Perspective (2010-2011 Yearbook, UAV International / Blyenburgh \& Co., France.

\section{Authors' Biographical Notes:}

Raid Al-Tahir holds a PhD. degree in Geodetic Sciences from The Ohio State University (USA) and a BSc. Engineering from the University of Baghdad (Iraq). Currently, he is a Senior Lecturer at the Department of Geomatics Engineering and Land Management, The University of the West Indies (Trinidad and Tobago). He is also an Adjunct Professor at the Department of Geodesy and Geomatics Engineering, the University of New Brunswick (Canada). His research interests include spatial data collection, quantitative analysis, and modelling using geo-spatial images and Geographic Information System to address issues related to land cover dynamics with implications for environmental degradation and natural resource management.

Giatri K. Lalla obtained a BSc degree in Geomatics and is pursuing a MSc degree in Geoinformatics from The University of the West Indies (Trinidad and Tobago). She has been employed within the Trinidad and Tobago Civil Aviation Authority for 3 years and it is where she specialised in Unmanned Aircraft Systems thus in 2020, she was appointed as the organization's Unmanned Aircraft Systems Officer. Ms. Lalla holds a Federal Aviation Administration Part 107 Remote Pilot's License. She has obtained professional education on the subject of Unmanned Aircraft Systems from Embry-Riddle Aeronautical University (USA). She continues to expand her knowledge on Unmanned Aircraft Systems, general aviation and geographic information systems. 